
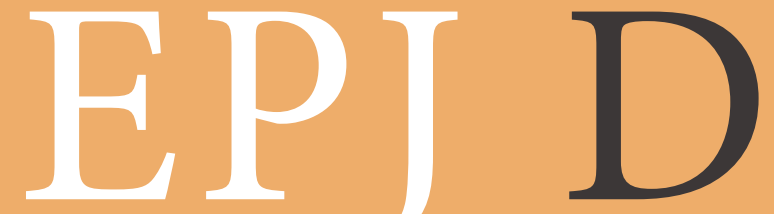

Atomic, Molecular,

Optical and Plasma Physics EPJ.ors

Eur. Phys. J. D (2017) 71: 257

DOI: $10.1140 /$ epjd/e2017-80167-9

Multi-longitudinal-mode micro-laser model

Kestutis Staliunas
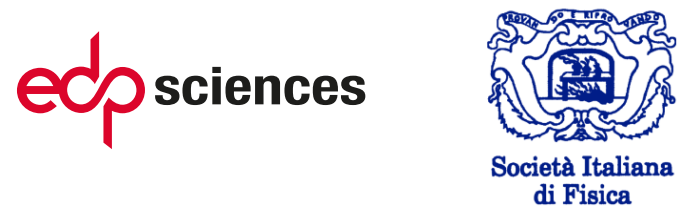

望 Springer 


\title{
Multi-longitudinal-mode micro-laser model ${ }^{\star}$
}

\author{
Kestutis Staliunas ${ }^{\mathrm{a}}$
}

ICREA, Departament de Física, Universitat Politècnica de Catalunya, Rambla Sant Nebridi, 22, 08222 Terrassa, Barcelona, Spain

Received 12 March 2017 / Received in final form 12 June 2017

Published online 19 October 2017 - (c) EDP Sciences, Società Italiana di Fisica, Springer-Verlag 2017

\begin{abstract}
We derive a convenient model for broad aperture micro-lasers, such as microchip lasers, broad area semiconductor lasers, or VCSELs, taking into account several longitudinal mode families. We provide linear stability analysis, and show characteristic spatio-temporal dynamics in such multi-longitudinal mode laser models. Moreover, we derive the coupled mode model in the presence of intracavity refraction index modulation (intracavity photonic crystal).
\end{abstract}

\section{Introduction}

Transverse pattern formation in broad area lasers and laser like systems (nonlinear resonators) gained a huge popularity since late 80 s and early 90 s (see the pioneering works [1-13], as well as more recent reviews [14,15]). The numerical studies in the most of "laser pattern forming" studies were performed on the basis on the single-longitudinal mode family Maxwell-Bloch Equations (MBE):

$$
\begin{aligned}
& \frac{\partial E}{\partial t}=\left[-(1+i \omega)+i d \nabla^{2}+i V\left(\mathbf{r}_{\perp}\right)\right] E+P \\
& \frac{\partial P}{\partial t}=-\gamma_{\perp}[P+E D] \\
& \frac{\partial D}{\partial t}=-\gamma_{\|}\left(D-D_{0}\left(\mathbf{r}_{\perp}\right)+\frac{E P^{*}+E^{*} P}{2}\right)
\end{aligned}
$$

Here the complex-valued fields $E\left(\mathbf{r}_{\perp}, t\right)$ and $P\left(\mathbf{r}_{\perp}, t\right)$ are the envelopes of the electromagnetic (optical) field and material polarization, defined in space $\mathbf{r}_{\perp}=(x, y)$ perpendicular to the optical axis of the laser, and evolving in time t. $D\left(\mathbf{r}_{\perp}, t\right)$ is the real-valued distributions of the population inversion which, in the absence of the field in the resonator, equals to the pump distribution $D_{0}\left(\mathbf{r}_{\perp}\right) \cdot \gamma_{\perp}$ and $\gamma_{\|}$ are the decay rates of the polarization and population inversion respectively normalized to photon decay rate. $\omega_{0}$ is the resonator detuning (the resonator resonance frequency with respect to the center of the gain line) normalized to the photon decay rate as well. The time is normalized to photon lifetime $\tau=L f / c, L$ is the full (roundtrip) length of the resonator, and $f$ is the cavity finesse. $V\left(\mathbf{r}_{\perp}\right)$ is the "trap potential" for the light due to the curved (parabolic)

\footnotetext{
* Contribution to the Topical Issue "Theory and Applications of the Lugiato-Lefever Equation", edited by Yanne K. Chembo, Damia Gomila, Mustapha Tlidi, Curtis R. Menyuk.

a e-mail: kestutis.staliunas@icrea.cat
}

cavity mirrors, or in case of planar cavity micro-laser, due to thermal lensing, $V\left(\mathbf{r}_{\perp}\right)=\beta D_{0}\left(\mathbf{r}_{\perp}\right) . d$ is the diffraction constant which can be expressed through the parameters of the resonator: $d=L \lambda f /(4 \pi)$.

The system (1) is the most basic one: in real situations it includes extra ingredients. For instance for semiconductor laser (1) contains a frequency shift of polarization depending on the inversion (the linewidth enhancement factor $\alpha_{H}$ ) [16,17]. The (1) can be supplemented by intracavity saturable absorption, or by intracavity Kerr media, depending on the situation [18-20].

Another widespread model is the Lugiato-Lefever model [2], which could be derived from the MBE (1) in a "passive limit" $D_{0}\left(\mathbf{r}_{\perp}\right) \rightarrow 0$, including linewidth enhancement factor $\alpha_{H}$, and adding an external drive. The Lugiato-Lefever model is also used to describe a variety of dynamical phenomenon, like spatial soliton formation [21,22], formation of extended passive patterns [6-8], spatial "rocking" [23], and many others, which is also a single-longitudinal-mode model. Multi-longitudinal-mode models presented below in this article are applicable to Lugiato-Lefever model as well.

The electromagnetic part of the MBE, the (1a), is derived from the field equation:

$$
\frac{1}{c^{2}} \frac{\partial^{2} E}{\partial t^{2}}-\nabla^{2} E=\varepsilon_{0} \mu_{0} \frac{\partial^{2} P}{\partial t^{2}},
$$

where $c=\left(\varepsilon_{0} \mu_{0}\right)^{-1 / 2}$ is the speed of light. To derive (1) the field $E(\mathbf{r}, t)$ and the material polarization $P(\mathbf{r}, t)$ is expressed in terms of slowly varying envelopes in transverse space: $E(\mathbf{r}, t)=\exp \left(i \omega_{0} t\right) \exp (2 \pi i m z / L) E\left(\mathbf{r}_{\perp}, t\right)+$ c.c., and the limit of fast carrier oscillations is considered: $\omega_{0}|E| \gg|\partial E / \partial t|, \omega_{0}|P| \gg|\partial P / \partial t|, k_{0}|E| \gg|\nabla E|$ to eliminate the space-time derivatives except for those of lowest (dominating) order. The transverse coordinate $\mathbf{r}_{\perp}$ spans the two-dimensional space transverse to the optical axis (the diffraction term is $i d \nabla_{\perp}^{2}=i d\left(\partial^{2} / \partial x^{2}+\partial^{2} / \partial y^{2}\right)$ ), 
a)

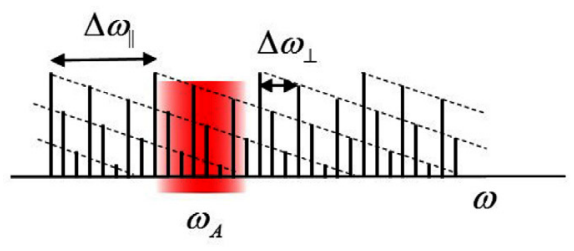

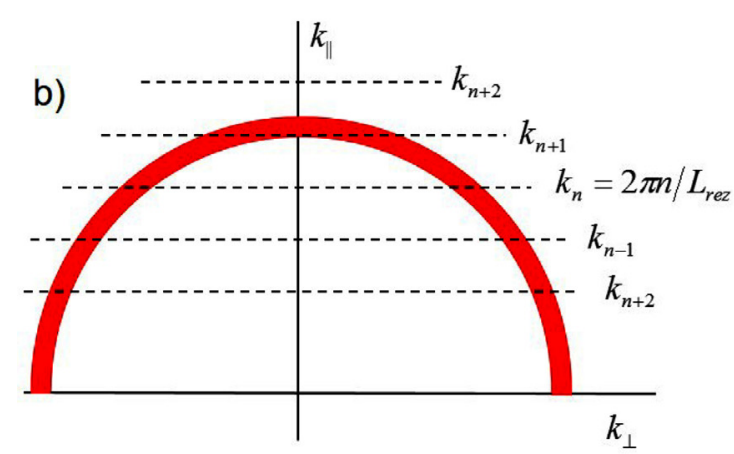

Fig. 1. (a) Double-comb of frequencies for multi-longitudinal multi-transversal mode lasers: the longitudinal mode separation is $\Delta \omega_{\|}$and transverse mode separation is $\Delta \omega_{\perp}$. Longitudinal mode families are indicated by inclined dashed lines. (b) Alternative interpretation of the mode structure of multimode laser: the inter- section of the longitudinal modes (dashed lines, or corresponding planes with $k_{\|}=2 P n / L$ ) results in resonant transverse wavenumbers (tilted waves), or in Fresnel rings in case of two transverse dimensions.

and the longitudinal dependence of the fields is not taken into account. Therefore the (1) describes the lasers with single-longitudinal mode family, i.e. the two-dimensional field distributions slowly evolving in time.

The single longitudinal-mode MBE (1), although thousands of times used in literature, is, strictly speaking, not applicable for simulations of real lasers. A single exception is perhaps the Vertical Cavity Surface Emitting Laser (VCSELS), where the cavity is so short (of the order of single wavelength), that only one longitudinal mode family exists. The modifications of the model (1) were successfully applied for a study of various cavity-soliton formation regimes in VCSELS $[16,17]$. However for the other types of lasers, even for the micro-lasers, like the microchips or broad area semiconductor diodes (edge emitting lasers), where the resonator length is typically of order of millimeter, the resonator supports thousands of longitudinal mode families, and the MBE model (1) is not applicable in a strict sense. On the other extreme, the fiber lasers, typically of meter- and kilometer length resonators (see e.g. revue [24]), is completely out of the scope of applicability of the single-longitudinal-mode MBE models (1).

Sometimes it is erroneously assumed that a very narrow gain line (small values of $\gamma_{\perp}$ ) can justify the validity of the single longitudinal mode model (1). For instance if the gain line is narrower than the free spectral range (the frequency separation of the longitudinal modes), $\gamma_{\perp} \leqslant \Delta \omega_{\perp}$, which for instance is the case in typical gas-lasers, then the single longitudinal mode approach is considered legitimate. This is correct only for the case of also single, or very few, transverse modes (for instance if the higher order transverse modes are suppressed by the aperture in the resonator), however it is incorrect for the broad-aperture lasers. Figure 1 illustrates the situation. Each longitudinal mode family contains many transverse modes (which degenerates into a continuum of modes in in the case of infinitely broad aperture), and the longitudinal mode families overlap in frequency domain. I.e., as follows from the Figure 1, even extremely narrow gain line can support simultaneous emission on several longitudinal mode families.

The situation illustrated in Figure 1 is typical for many micro-lasers. Therefore for a correct description of the field dynamics in such lasers, the single mode MBE model (1) is to be revisited.

One possibility to solve the problem is the use more complete models, where the field propagation along the resonator is explicitly considered. Technically the time evolution operator in (1a) is to be substituted by $\partial / \partial t \rightarrow$ $\partial / \partial t \pm c \partial / \partial z$ for the forward-backward propagating field components, and the boundary conditions are to be considered to link the forward-backward fields on the cavity mirrors. The round-trip model showed itself to be successful to simulate the fields in broad area edge-emitting micro-lasers $[25,26]$, where only one transverse coordinate (and of course longitudinal coordinate) is relevant. However for the fully 3D lasers, like for instance microchip lasers, where one should consider the field distributions in both transverse directions, the full MBE simulations are hardly possible (with some small exceptions, for instance [27] for the passive nonlinear optical systems). Moreover, the round-trip models, because of their structural complexity, do not allow analytical insights on the pattern formation.

Another approach is based on the introduction of field delays in the right side of MBE (1a) which in principle allows multi-longitudinal mode treatment $[28,29]$, however is also not very convenient from the viewpoint of numerical simulations, nor for the analytical insights.

Here we propose a relatively simple modification of MBE (1) for the multi-longitudinal mode lasers, such as microchips, extending it into the case of several longitudinal mode families. The model could be extremely useful for the lasers with spatially modulated cavities, i.e. for the cavities containing inside the photonic crystals [30,31], which up to now were simulated using complicated and time consuming models. 


\section{Multi-longitudinal mode MBE}

We expand the electromagnetic field into set of longitudinal mode families:

$$
\begin{aligned}
E(\mathbf{r}, t)= & \exp \left(i \omega_{0} t\right) \sum_{m} E_{m}(\mathbf{r}, t) \\
& \times \exp (2 \pi i m z / L) E\left(\mathbf{r}_{\perp}, t\right)+c . c .
\end{aligned}
$$

the complex envelopes of each field component depend only on transverse coordinates $\mathbf{r}_{\perp}=(x, y)$, but no more on the longitudinal coordinate. Generally, one could expand the polarization $P(\mathbf{r}, t)$ and the population inversion $D(\mathbf{r}, t)$ as well, in analogous way as $(3)$, which at the end results in a coupled equation system for each longitudinal mode component (see for instance [1]). This however does not reduce the complexity of the problem: instead of explicit dependence of the fields on $z$-coordinate one has just their expansions in Fourier series. However, for the case of typical microchip lasers the amplifying media is a thin slice, significantly thinner than the length of the resonator, the material fields (polarization, population inversion) can be considered as two-dimensional functions within this slice. Under the latest assumption, the MBE can be written in a relative simple way:

$$
\begin{aligned}
\frac{\partial E_{m}}{\partial t} & =\left[-\left(1+i \omega_{m}\right)+i d \nabla^{2}+i V\left(\mathbf{r}_{\perp}\right)\right] E_{m}+P, \\
\frac{\partial P}{\partial t} & =-\gamma_{\perp}[P+E D], \\
\frac{\partial D}{\partial t} & =-\gamma_{\|}\left(D-D_{0}+\frac{E P^{*}+E^{*} P}{2}\right),
\end{aligned}
$$

with $E(\mathbf{r}, t)=\sum_{m} E_{m}\left(\mathbf{r}_{\perp}, t\right)$, which is simply the expansion (3), choosing without the loss of generality the reference coordinate $z=0$ on the narrow slice of the active media. Note, that in (4a) each field component contains a different detuning, $\omega_{m}=\omega_{0}+m \Delta \omega_{\perp}$, therefore the (4) cannot be further simplified by simple addition of the field components in (4a).

The rest of the article is devoted to the study of (4) for the case of several longitudinal mode families. In some situations, the dynamics of multi-longitudinal-mode case does not lead to principal differences from single longitudinal mode dynamics (where only the nearest-to-axis transverse modes are considered), in particular in cases when the radiation on other longitudinal mode families is suppressed, however in some cases the multimode effects are important. The multi-longitudinal mode effects are especially important in case of intracavity scattering between different mode families, due to for instance intracavity photonic crystal, or the intracavity diffraction grating $[30,31]$. The latter situation is shortly considered in the concluding part of the article.

\section{Important simplifications}

In many relevant cases the (4) allows convenient simplifications. One important class of the lasers is when the polarization is fast compared with the relaxation of the optical field, and population inversion: $\gamma_{\perp} \gg 1, \gamma_{\|}$. For instance semiconductor lasers fell into that class. In this case polarization can be adiabatically eliminated from (4b), $\frac{\partial P}{\partial t}=-\gamma_{\perp}[P+E D]$, leading to:

$$
\begin{aligned}
\frac{\partial E_{m}}{\partial t} & =\left[-\left(1+i \omega_{m}\right)+i d \nabla^{2}+i V\left(\mathbf{r}_{\perp}\right)\right] E_{m}+E D \\
\frac{\partial D}{\partial t} & =-\gamma_{\|}\left(D-D_{0}+D|E|^{2}\right)
\end{aligned}
$$

Here as above, $E(\mathbf{r}, t)=\sum_{m} E_{m}\left(\mathbf{r}_{\perp}, t\right)$.

Another important limit is the class-A laser, when the relaxation of population inversion is also fast, $\gamma_{\perp}, \gamma_{||} \gg 1$. Then (4) and (5) further reduces to:

$$
\frac{\partial E_{m}}{\partial t}=\left[-\left(1+i \omega_{m}\right)+i d \nabla^{2}+i V\left(\mathbf{r}_{\perp}\right)\right] E_{m}+\frac{E D_{0}}{1+|E|^{2}}
$$

or in case of cubic approximation: $1 /\left(1+|E|^{2}\right) \approx 1-|E|^{2}$ valid close to the lasing threshold, $\left(D_{0}-1\right) \ll 1$ :

$$
\frac{\partial E_{m}}{\partial t}=\left[-\left(1+i \omega_{m}\right)+i d \nabla^{2}+i V\left(\mathbf{r}_{\perp}\right)\right] E_{m}+D_{0} E-E|E|^{2}
$$

which is an analog of the Complex Ginzburg-Landau Equation (CGLE) derived for a single longitudinal mode laser $[4,6,9,10]$. Here (7) comprises a system of CGLEs mutually coupled through the term $D_{0} E=D_{0} \sum E_{m}$ as well through the saturating nonlinearity $E|E|^{2}=$ $\left(\sum E_{m}\right)^{2} \sum E_{m}^{*}$. Each of the CGLEs in a system has different (equidistantly distributed) detunings $\omega_{m}=\omega_{0}+$ $m \Delta \omega_{\|}$, like in original multi-longitudinal mode MBE (4).

Note, that the multi-longitudinal mode CGLE (7) in the passive cavity limit $D_{0} \rightarrow 0$, with additional injection term, could be called by multi-longitudinal mode LugiatoLefever model.

\section{Linear stability analysis}

We consider the zero solution of (4a), (4b): $E_{m}=0$, $P=0, D=D_{0}$ (the point of the switching-on of a laser), and perturb it by the modes of spatial modulation: $E_{m}=e_{m} \exp (i k x), P=p \exp (i k x), e_{m}, p \ll 1$. The evolution matrix for the perturbation modes in (4) reads:

$$
\begin{aligned}
& \hat{M}= \\
& {\left[\begin{array}{ccclc}
-\gamma_{\perp} & \gamma_{\perp} D_{0} & \gamma_{\perp} D_{0} & \ldots & \gamma_{\perp} D_{0} \\
1 & -1-i \omega_{1}-i d k^{2} & 0 & \ldots & 0 \\
1 & 0 & -1-i \omega_{2}-i d k^{2} & \ldots & 0 \\
\ldots & \ldots & \ldots & \ldots & \ldots \\
1 & 0 & 0 & \ldots & -1-i \omega_{N}-i d k^{2}
\end{array}\right],}
\end{aligned}
$$

which is a matrix of rank $(N+1)$ for the $N$ longitudinal mode families. Note that the (4c) decouples from the rest of (4), and the corresponding variable does not appear in (8), i.e. the rank is not $(N+2)$. The perturbation column 


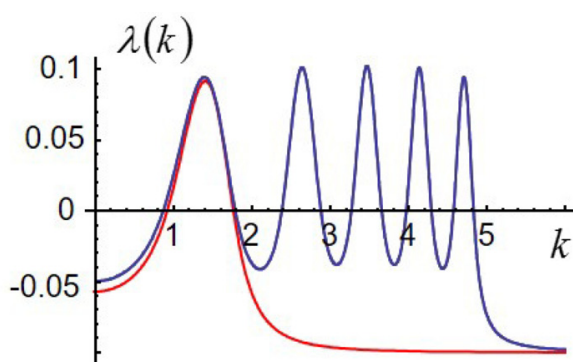

Fig. 2. Linear stability analysis showing the multiple instability areas corresponding to multiple longitudinal mode families. The calculations for single mode lasers at the same detuning is shown for comparison by red curve. Parameters used: $d=1$, $\omega_{0}=2, \Delta \omega_{\|}=5, D_{0}=2, \gamma_{\perp}=0.1$.

vector is $\left(p, e_{1}, e_{2}, \ldots, e_{n}\right)^{T}$. Calculation of the eigenvalues of (8) leads to the following implicit expression:

$$
\sum_{m=1, N} \frac{\gamma_{\perp} D_{0}}{\left(1+\lambda+i \omega_{m}+i d k^{2}\right)}-\gamma_{\perp}-\lambda=0
$$

The eigenvalues can be calculated numerically solving the (9), which leads directly to the multiple instability areas (see Fig. 2), which are compatible with the initial intuition, illustrated in Figure 1.

\section{Numerics}

First, we simulated an idealized case of infinitely large aspect ratio laser without flat cavity mirrors, and with homogeneous pump profile. The radiation then fills the complete transverse space. Technically, it is convenient to use periodic boundary conditions in the lateral direction, which naturally occur due to discrete Fourier space in split-step method used.

Such numerical simulations first at all reproduce the multiple instability areas, following from the linear stability analysis. We simulate the 2D case, and the collection of results is presented in Figure 3. In particular, the multiple rings are visible in the far fields in Figure 3, where we show the snapshots of far field in a transient stage for the amplifying noise above the generation threshold.

Most frequently the final nonlinear patterns (the pump above the threshold, after the transients) show nothing spectacular with respect from single-longitudinal-mode model. Depending on the situation (the pump level, relaxation rates), one eventually obtains tilted wave patterns, with the tilt angle depending on the detuning [10], or square vortex lattices $[32,33]$. If in single longitudinal mode laser the different domains of tilted waves always belong to the same longitudinal mode family (they have different orientation), now sometimes the tilted waves from different domains sometimes can belong to different mode. Typically the tilted wave belonging to the most close to resonance longitudinal mode family survives and dominates the asymptotic regime. The scenarios in general is quite similar to the ones found in single-longitudinal mode models in this simplest case of infinitely extended "pure" laser [34].

In the case of parabolic resonator mirrors, which correspond to the trapping parabolic potential in transverse domain, the scenario is usually more involved. One can distinguish between the weakly nonlinear regimes occurring for the pump level close above the generation threshold, which essentially are the transverse modes or coupled states of modes of the cavity, Figure 4 the upper row. For the pump values stronger above the generation threshold one observes the "essentially nonlinear" patterns, where the field distributions are different from the superposition of small number of transverse modes. The essentially nonlinear patterns are the domains of tilted waves, Figure 4, bottom row shows typical pictures of multi-longitudinal mode laser emission, where the transverse modes from different longitudinal mode families lock to the patterns dominated by tilted waves. In this case, due to symmetry a stationary concentric travelling wave pattern is obtained, or sometimes containing a vortex in the middle.

\section{Intracavity modulation}

Typically the multi-longitudinal-mode laser does not leads to spectacular differences from the single longitudinal mode cases, since the different longitudinal mode families compete, and most frequently finish with single longitudinal mode. In some cases, however, the multi-longitudinal mode study is more important, for instance, when the refraction index in the cavity is spatially modulated. Spatial modulation can couple the modes from the several longitudinal mode families, and in some cases, especially when the coupling is resonant, this can lead to essentially multilongitudinal-mode. We consider for simplicity a particular case of factorable modulation in transverse and longitudinal directions $\varepsilon(\mathbf{r})=\varepsilon_{\perp}\left(\mathbf{r}_{\perp}\right) \cdot \varepsilon_{\|}(z)$. In particular we consider periodic modulation in transverse space, and the arbitrary modulation along the resonator axis. In simplest case it could be considered as diffraction grating in the cavity.

Using the same expansion (3), the (4a) modifies to:

$$
\begin{aligned}
\frac{\partial E_{m}}{\partial t}=[ & \left.-(1+i \omega)+i d \nabla^{2}+i V\left(\mathbf{r}_{\perp}\right)\right] \\
& \times E_{m}+P+\frac{i \pi L f \varepsilon_{\perp}\left(\mathbf{r}_{\perp}\right)}{\lambda} \sum_{l} \Gamma_{l m} E_{l} .
\end{aligned}
$$

The last right side term is responsible for the coupling between the mode families, where the coupling coefficient matrix is given by:

$$
\Gamma_{l m}=\int A_{l}(z) \varepsilon_{\|}(z) A_{m}^{*}(z) d z
$$

where the longitudinal mode functions $A_{m}(z) \propto$ $\exp (i m 2 \pi z / L)$ of unperturbed resonator $\varepsilon_{\|}(z)=1$ are normalized so that $\Gamma_{l l}=1$. 

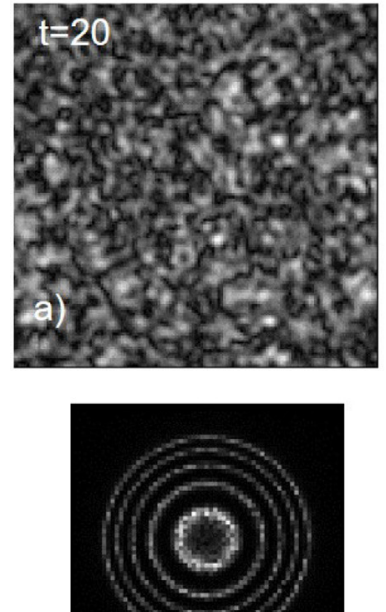

b)
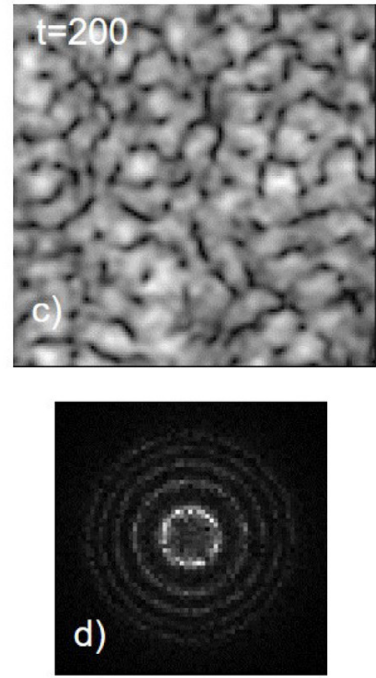
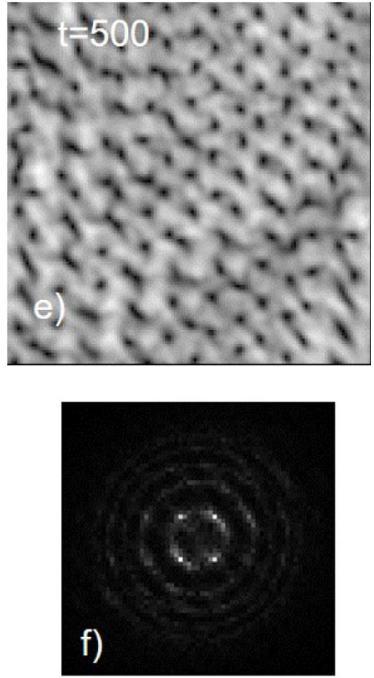

Fig. 3. Near-far field patterns (a), (c), (e) and their corresponding far field patterns (b), (d), (f) in different times in infinitely broad aspect-ratio lasers with flat cavity mirrors and homogeneous pump profile. The parameters as in Figure 2. Starting from a random distribution, the field evolves into multi-ring structure (five rings, as five longitudinal modes were considered). In the course of evolution the highest longitudinal mode (the ring of smallest radio) survives. The integration was performed on a grid $(128 \times 128)$ with the size of integration region 32 with $d=1$.
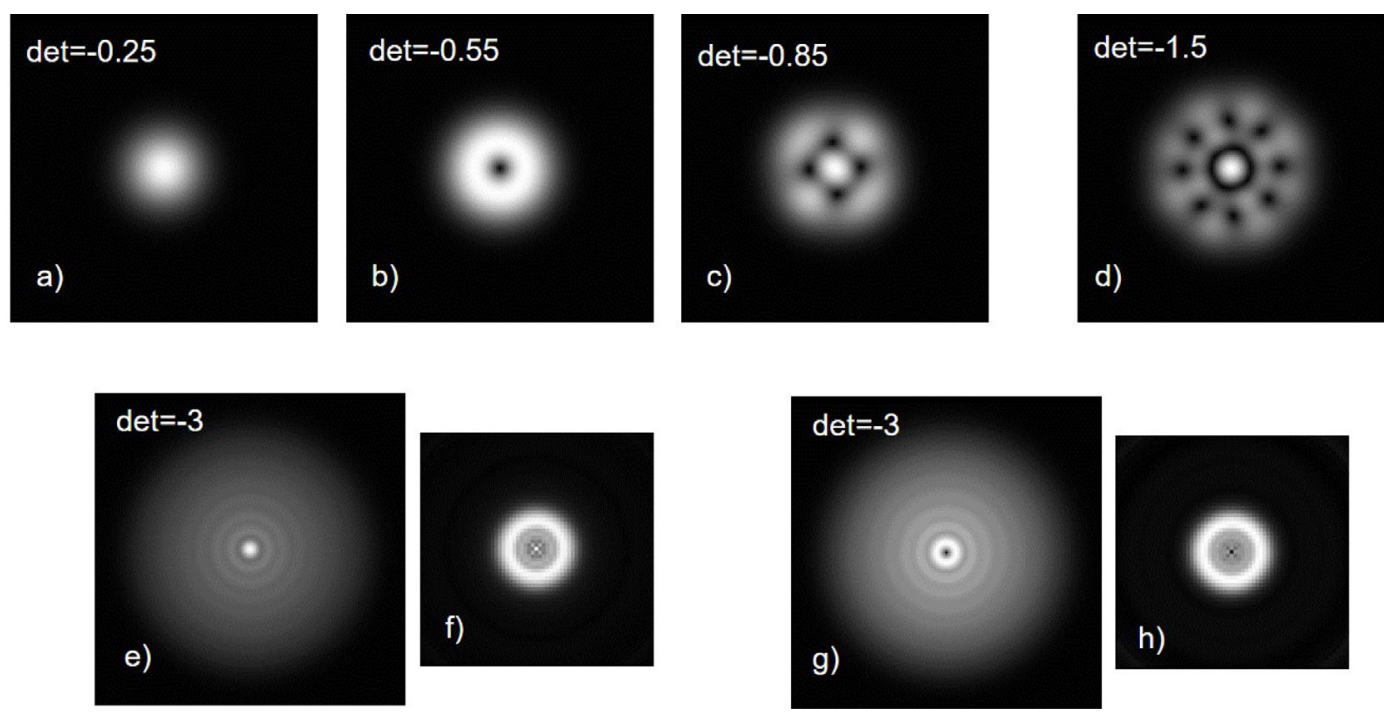

Fig. 4. Patterns for multi-mode lasers with parabolic mirrors. (a-d) Near threshold patterns (at different detunings), which are identic with the corresponding patterns in single-longitudinal mode laser. The row is essentially single transverse modes (a, b), or combinations of modes belonging to degenerated higher order transverse mode families. (e, g) Together with corresponding far field distributions ( $\mathrm{f}, \mathrm{h}$ ) are the patterns dominated by tilted waves at higher pump-above-threshold values. The parameters are as in Figure 3, except for the parabolic mirrors, which result in separation between transverse modes $\Delta \omega_{\perp}=0.3$.

The coupling matrix explicitly written has the structure:

$$
\Gamma_{l m}=\left[\begin{array}{ccccc}
\gamma_{0} & \gamma_{1} & \gamma_{2} & \gamma_{3} & \ldots \\
\gamma_{1}^{*} & \gamma_{0} & \gamma_{1} & \gamma_{2} & \ldots \\
\gamma_{2}^{*} & \gamma_{1}^{*} & \gamma_{0} & \gamma_{1} & \ldots \\
\gamma_{3}^{*} & \gamma_{2}^{*} & \gamma_{1}^{*} & \gamma_{0} & \ldots \\
\ldots & \ldots & \ldots & \ldots & \ldots
\end{array}\right]
$$

Note that the coupling matrix possess symmetries: $\Gamma_{l m}=$ $\Gamma_{m l}^{*}$ as $\varepsilon_{\| \mid}(z)$ is real valued, and $\Gamma_{l m}=\gamma_{l-m}$ (a multi diagonal-like structure). In limiting case, when the index is modulated on an infinitesimally thin plate $\varepsilon_{\|}(z)=1+$ $\Delta \varepsilon \cdot \delta(z)$ (thin diffraction grating), the matrix elements are equal $\Gamma_{i j}=\gamma_{i-j}=\Delta \varepsilon$ for all $i$ and $j$.

The detailed analysis of (10) is out of scope of the present article. It is provided here just with the purpose to demonstrate that the multi-longitudinal mode approach 
can be extended to more complicated cases of the modulated resonances.

\section{Conclusions}

In conclusion we have presented an extension of the singlelongitudinal-mode MBE model for the multi-longitudinalmode case. We proofed the derivation by calculation of basic cases: the linear stability analysis, and the simplest tilted-wave and mode patterns. We also provided the hierarchy of multi-longitudinal-mode models, starting from the full MBE, then simplifying it in the case of fast relaxation of polarization (e.g. semiconductor laser models), and finally to multi-longitudinal-mode CGLE and multilongitudinal-mode Lugiato-Lefever model in cases of classA lasers.

Finally, we proposed that the approach can be useful to treat the complicated cases when the intracavity material is modulated on a wavelength scale, which could result in coupling between the longitudinal and transverse modes. This would be a convenient model for a row of new phenomena in the latest situation (laser with intracavity photonic crystal), the situation inaccessible by singlelongitudinal mode models.

A financial support from NATO SPS Research Grant (SPS 985048), from Spanish Ministerio de Ciencia e Innovación, and European Union FEDER through Project FIS2015-65998-C21-P, and from European Union Horizon 2020 Framework EUROSTARS via Project E10524 HIP-Laser is acknowledged.

\section{References}

1. R. Graham, H. Haken, Z. Phys. 237, 31 (1970)

2. L.A. Lugiato, R. Lefever, Phys. Rev. Lett. 58, 2209 (1987)

3. L.A. Lugiato, C. Oldano, L.M. Narducci, JOSA B 5, 879 (1988)

4. P. Coullet, L. Gil, F. Rocca, Opt. Commun. 73, 403 (1989)

5. M. Brambilla, F. Battipede, L.A. Lugiato, V. Penna, F. Prati, C. Tamm, C.O. Weiss, Phys. Rev. A 43, 5090 (1991)

6. G.L. Oppo, G. D'Alessandro, W. Firth, Phys. Rev. A 44, $4712(1991)$

7. F.T. Arecchi, G. Giacomelli, P.L. Ramazza, S. Residori, Phys. Rev. Lett. 67, 3749 (1991)
8. S.A. Akhmanov, M.A. Vorontsov, V.Yu. Ivanov, A.V. Larichev, N.I. Zheleznik, JOSA B 9, 78 (1992)

9. K. Staliunas, Phys. Rev. A 48, 1573 (1993)

10. P.K. Jakobsen, J.V. Moloney, A.C. Newell, R. Indik, Phys. Rev. A 45, 8129 (1992)

11. M. Tlidi, M. Georghiou, P. Mandel, Phys. Rev. A 48, 4605 (1993)

12. Y.S. Kivshar, X. Yang, Chaos Solitons Fract. 4, 174 (1994)

13. W.J. Firth, C. Pare, Opt. Lett. 13, 1096 (1988)

14. K. Staliunas, V.J. Sanchez-Morcillo, Transverse Patterns in Nonlinear Optical Resonators, Springer Tracts in Modern Physics (Springer Verlag, 2003), Vol. 183

15. M. Tlidi, K. Staliunas, K. Panajotov, A.G. Vladimirov, M.G. Clerc, Phil. Trans. R. Soc. A 372, 20140101 (2014)

16. D. Michaelis, U. Peschel, F. Lederer, Phys. Rev. A 56, R3366 (1997)

17. L. Spinelli, G. Tissoni, M. Brambilla, F. Prati, L.A. Lugiato, Phys. Rev. A 58, 2542 (1998)

18. S. Fauve, O. Thual, Phys. Rev. Lett. 64, 282 (1990)

19. N.N. Rosanov, in Progress in Optics, edited by E. Wolf (North-Holland, Amsterdam, 1996), Vol. XXXV

20. K. Staliunas, V.B. Taranenko, G. Slekys, R. Viselga, C.O. Weiss, Phys. Rev. A 57, 599 (1998)

21. A.J. Scroggie, W.J. Firth, G.S. McDonald, M. Tlidi, R. Lefever, L.A. Lugiato, Chaos Solitons Fract. 4, 1323 (1994)

22. G. Slekys, K. Staliunas, C.O. Weiss, Opt. Commun. 149, 113 (1998)

23. G.J. de Valcárcel, K. Staliunas, Phys. Rev. A 87, 043802 (2013)

24. S.K. Turitsyn, B.G. Bale, M.P. Fedoruk, Phys. Rep. 521, $135(2012)$

25. M. Radziunas, K. Staliunas, EPL 95, 14002 (2011)

26. R. Herrero, M. Botey, M. Radziunas, K. Staliunas, Opt. Lett. 37, 5253 (2012)

27. K. Staliunas, Phys. Rev. Lett. 81, 81 (1998)

28. P Khandokhin, Ya. Khanin, C. Celet, D. Dangoisse, P. Glorieux, Opt. Commun. 123, 372 (1996)

29. G. Kozyreff, A.G. Vladimirov, P. Mandel, Phys. Rev. Lett. 85, 3809 (2000)

30. K. Staliunas, M. Peckus, V. Sirutkaitis, Phys. Rev. A 76, 051803 (2007)

31. K. Staliunas, O. Egorov, Yu.S. Kivshar, F. Lederer, Phys. Rev. Lett. 101, 153903 (2008)

32. K. Staliunas, C.O. Weiss, Physica D 81, 79 (1995)

33. K. Staliunas, C.O. Weiss, J. Opt. Soc. Am. B 12, 1142 (1995)

34. K. Staliunas, G. Slekys, C.O. Weiss, Phys. Rev. Lett. 79, 2658 (1997) 